\title{
The Application of Context Theory in English Teaching of Reading
}

\author{
Jiang Zhu \\ School of Foreign Languages, Changchun University of Science and Technology \\ Changchun 130022, China
}

Tel: 86-431-8558-3033Ｅ-mail: zhujiang98@163.com

Lemeng Han

Department of Common Foreign Language Teaching, Guanghua College of Changchun University

Changchun 130117, China

E-mail: hanlemeng1982@sina.com

The research is financed by “Jilin Province Social Science Fund Project”, Item Code: $2009 B 148$.

\begin{abstract}
Context theory is a very important theory in English teaching, especially the teaching of reading. This paper first analyzes the theory of context, including the features of context and some principles in context theory. Then the paper discusses the application of context theory in English teaching of reading, including some problems met in reading comprehension test and some teaching methods related to context theory.
\end{abstract}

Keywords: Context, Co-text, Local interpretation, Analogy, Prediction

\section{Introduction}

In the production of text, the producer will put the text in the restriction of certain society, language and matter world. The society, language and matter world that interact with the text are called context. According to Malinowski, context is classified into three types: context of utterance, context of situation, and context of culture. These elements are quite important in analysis of the text. For example, in order to understand a piece of text, it is necessary to know whom the speaker and hearer are, and the time and place of the production of the text. In this paper I shall discuss the theory of context and its application in English teaching of reading.

\section{Features of Context}

J. R. Firth, regarded by many as the founder of modern British linguistics, proposed an approach to the principled description of social contexts which bears a close resemblance to more recent descriptions which we shall examine:

"My view was, and still is, that 'context of situation' is best used as a suitable schematic construct to apply to language events... A context of situation for linguistic work brings into relation the following categories:

A. The relevant features of participants: persons, personalities.

(i) The verbal action of the participants.

(ii) The non-verbal action of the participants.

B. The relevant objects.

C. The effect of speech act."

An approach similarly emphasizing the importance of an ethnographic view of communicative events within communities has been developed by Hymes in a series of articles. Hymes views the role of context as limiting the range of possible interpretations on the one hand and as supporting the intended interpretation on the other hand.

Hymes sets about specifying the features of context which may be relevant to the identification of a type of speech event in a way reminiscent of Firth's. Like Firth, he seizes first on the "persons" participating in the speech event. Generalizing over speech events, he abstracts the roles "addressor" and "addressee". The addressor is the speaker or writer who produces the utterance. The addressee is the hearer or reader who is the recipient of the utterance. Knowledge of the addressor in a given communicative event makes it possible for the analyst to imagine what that particular person is likely to say. Knowledge of his addressee constrains the analyst's expectations even further. Thus, if you know the speaker is the prime minister or the departmental secretary or your family doctor or your mother, or if you know that the speaker is speaking to a colleague or his bank manager or a small child, you will have different 
expectations of the sort of language which will be produced, both with respect to form and to content. If you know, further, what is being talked about, Hymes' category of "topic", your expectations will be further constrained. If then you have information about the "setting", both in terms of where the event is situated, and in terms of the physical relations of the persons with respect to posture and gesture and facial expression, your expectations will be still further limited.

The remaining features of context which Hymes discusses include large-scale features like channel (how is contact between the participants in the event being maintained — by speech, writing, signing, smoke signals), lode (what language, or dialect, or style of language is being used), message form (what form is intended — chat, debate, sermon, fairy-tale, sonnet, love-letter, etc.) and event (the nature of the communicative event within which a genre may be embedded). Later Hymes adds other features, for example key (which involves evaluation), and purpose (what the participants intended should come about as a result of the communicative event).

\section{Co-text}

So far we have mainly discussed the physical context in which single utterances are embedded and we have paid rather little attention to the previous text co-ordinate. Lewis (1972) introduced the co-ordinate to take account of sentences that include specific reference to what has been mentioned before as in phrases like the aforementioned. However, it is the case that any sentence other than the first in a fragment of text will have the whole of its interpretation forcibly constrained by the preceding text, not just those phrases which obviously and specifically refer to the preceding text, like the aforementioned. The words that occur in text are constrained by what we call their co-text. The interpretation of utterances within a text is also constrained by co-text. Co-text refers to the relations between different parts of the text. Now consider the following text:

(1a)A man and a woman are sitting in the living room. The woman is sitting reading quite happily. The man is bored and goes to the window. He looks out of the window and gets himself ready and goes out.

The reader must interpret "the woman is sitting reading quite happily" as the "woman" already mentioned, hence must construct an interpretation which has her "sitting reading quite happily in the living room". Similarly the "window" which the man approaches must be interpreted as "the window of the living room". The speaker continues with a change of location and we have to assume that what follows is within the newly introduced location:

(1b) He goes to a club. He has a drink and talks to the barman. Then he starts dancing with a beautiful girl and has a good time.

We interpret everything that happens here as happening to the man we met in the living room who is now at "a club". So he "has a drink, talks to the barman, starts dancing and has a good time" all at the "club". The speaker announces another change of location:

(1c) Then he goes home and calls her. His wife overhears him.

Again we assume that we are still talking about the same man. He has returned "home" where the "living room" we first meet is located. Now the analyst may be doubtful on how to interpret the sentence "and calls her", for the man might reasonably go into the house and call (shout for) his wife. However, this interpretation is ruled out by the following co-text "and his wife overhears him". So we are obliged to interpret "calls" as meaning "phones" and "her" as "the beautiful girl with whom he danced".

For the moment the main point we are concerned to make is to stress the power of co-text in constraining interpretation. Even in the absence of information about place and time of original utterance, even in the absence of information about the speaker/writer and his intended recipient, it is often possible to reconstruct at least some part of the physical context and to arrive at some interpretation of the text. In general, the more co-text there is, the more secure the interpretation is. Text creates its own context. As Izard (1975) remarks: "Communications do not merely depend on the context for their interpretation, they change that context."

\section{The Principle of "Local Interpretation"}

When people read and understand a text, they will use some principles to make sure that they will understand the text reasonably. One principle is called the principle of local interpretation. This principle instructs the hearer not to construct a context any larger than he needs to arrive at an interpretation. Now let us see how we understand Sack's (1972) much-quoted sequence with this principle:

(2) The baby cried.

The mommy packed it up.

It is possible to imagine that the first of these sentences describes one event and the second describes another quite unrelated event. However, the principle of local interpretation will guide us to construct a limited context in which "the mommy" is the mentioned baby's mother and the expression "it" is used to refer to the previously mentioned baby. 
Moreover the sequence of events will be understood as happening adjacently in time and situated adjacently in place. It does not even occur to the reader that the baby might have cried one year in China and be picked up by its mother a year later in America. It would be possible to establish a setting in which such a sequence of events would be plausible, but, if no such setting is established, the reader will assume a local interpretation in respect of time, place and participants.

It must be obvious that "local interpretation" may only be vaguely conceptualized. It seems unlikely that in interpreting (2) the reader postulates any exact physical distance between the mother and the baby at the point before the mother picks the child up, or that he bothers to wonder whether the mother picks the child up after it has finished crying or whether the child was still crying when the mother picked it up. Similarly it seems unlikely that the reader will bother to construct a three-dimensional, photographic representation of "the baby" who cries in the first sentence and who is picked up in the second sentence. "Local interpretation" probably relates to another strategy which instructs the hearer or reader to do as little processing as possible, only to construct a representation which is sufficiently specific to permit an interpretation which is adequate for what the hearer judges the purpose of the utterance to be.

Everything that I have said so far in this section leans heavily on the hearer's or reader's ability to utilize his knowledge of the world and his past experience of similar events in interpreting the language which he encounters. It is the experience of similar events that enables him to judge what the purpose of an utterance might be. It is his knowledge of the world that constrains his local interpretation.

\section{The Principle of "Analogy"}

Another principle which people use in reading and understanding a text is the principle of analogy. This principle will provide a reasonably secure framework for interpretation for the hearers and for the analyst most of the time. Most of the time, things will indeed conform to our expectations. However, conventions can be flouted and expectations upset, either deliberately for a stylistic effect, or by accident or oversight. When the speaker or writer is deliberately flouting a convention or upsetting an expectation for a stylistic effect, he can only bring off that effect because the convention or expectation exists.

The principle of analogy is one of the fundamental heuristics which hearers and analysts adopt in determining interpretations in context. They assume that everything will remain as it was before unless they are given specific notice that some aspect has changed.

\section{The Application of Cultural Context}

The usage of language depends on context. Any text is the product of certain context. Reading is the process of understanding text. If the reader cannot understand the context with which the writer produces the text correctly, he cannot understand the text according to the writer's intention. Situation, common knowledge and the subjective factors of the participants are essential conditions of understanding text. Steffensen (1986) asked Indian students who speak English and American students to read a text which reflects Indian culture. After reading he asked the students to recall the plot. He found that in the understanding of the third "they" in the text, the Indian students whose linguistic competences were relatively weak could make correct judgments, while the American students whose linguistic competences were strong could not:

(3) Prema's parents were not sure how they felt about that, but they allowed him to see her anyway. In this day and age they were lucky.

According to traditional Indian custom, the fiancé cannot see the fiancée before wedding ceremony. If the fiancé wants to see the fiancée before wedding ceremony, he must get the permission of the fiancée's parents. With the development of society, young people seldom obey this custom. When Prema's fiancé requested to see her before wedding ceremony, Prema's parents knew that their future son-in-law behaved himself, obeyed the tradition and respected them. They felt lucky to have such a son-in-law. American students did not know this kind of culture. They believed that the fiancé and fiancée were unfortunate because they could not see each other until wedding ceremony. Now they were allowed to see each other. They were very lucky. So the American students believed that "they" referred to Prema and her fiancé. This example shows us the role of cultural context or common knowledge in reading.

\section{Problems Met in Reading Comprehension Test}

Reading comprehension test can check the students' ability and effect of understanding the language. In such kind of test the students should know the context that the writer constructs and they should construct a context that helps them understand the text. This will greatly affect the students' accuracy and speed in the test. Many students only notice the surface meaning of words and sentences. They do not enter the context positively and do not stimulate the common knowledge with the writer. When these students meet several unknown words, they will feel quite helpless. Now let us look at the following reading comprehension test questions and analyze how to get correct answers to these questions.

(4)A team of researchers has found that immunizing patients with bee venom instead of with the bees' crushed bodies can better prevent serious and sometimes fatal sting reactions in the more than one million Americans who are 
hypersensitive to bee stings. The crushed-body treatment has been standard for fifty years, but a report released recently said that it was ineffective. The serum made from the crushed bodies of bees produced more adverse reactions than the injections of the venom did.

The research compared results of the crushed-body treatment with results of immunotherapy that used insects' venom and also with results of a placebo. After six to ten weeks of immunization, allergic reactions to stings occurred in seven of twelve patients treated with the placebo, seven of twelve treated with crushed-body extract, and one of eighteen treated with the venom.

Question 1: What is the main topic of the passage?

A. A new treatment for people allergic to bee stings.

B. A more effective method of preventing bee stings.

C. The use of placebo in treating hypersensitive patients.

D. Bee venom causing fatal reactions in hypersensitive patients.

Question 2: The most successful treatment described in the passage was a serum prepared from

A. the blood of patients who had been stung

B. poison extracted from bees

C. crushed bodies of bees

D. a placebo and a crushed-body extract

Question 3: In order to be successful, the treatment referred to in the passage must be administered

A. by a series of injections given before the patient is exposed

B. by injections immediately after the patient has been stung

C. orally for six to ten weeks before the patient is stung

D. orally immediately after the patient is stung

There are some rarely used words, e.g. venom, serum, immunotherapy, adverse reaction, placebo, allergic reaction, etc. in the text. If the teacher pays little attention to the role of context in everyday teaching, the students will be puzzled by the unknown words and spend a lot of time guessing the meaning of these words. Thus the speed of comprehension is affected and the students cannot get the answers to the questions in the limited time. On the contrary, if the students get training of this aspect in their study, comprehension will not be affected by the unknown words.

\section{The Method of Prediction in Reading Comprehension Test}

In 1985 Halliday and Hasan pointed out that a learner of English language should learn to make correct prediction. "Prediction" here means putting forward such question as "What will appear in the following part?" and answering the question. According to the theory of "register", field of discourse is used to predict experiential meaning, tenor of discourse is used to predict interpersonal meaning, and mode of discourse is used to predict textual meaning. Although there are some unknown words, we can make the following location after reading the first sentence of example (4):

Field of discourse: Discussing the prevention of sting reactions

Tenor of discourse: Doctor or scientist communicates with the reader

Mode of discourse: Expository written report

By this location, we can make global prediction to the text. In this text the doctor or scientist reports in written form the prevention of sting reactions to us. The unknown words must be medical terminologies. Then we make local prediction:

Researchers found that (a) immunizing patients with venom can prevent serious and sometime fatal sting reactions better than (b) immunizing with bees' crushed bodies.

According to experience, we predict that the following part will make comment on (a) and (b):

(b) has been standard for fifty years, but a report released recently said that it was ineffective.

Experience shows that the following part will discuss why the effect is not good:

(b) produced more adverse reactions than (a).

We predict that the writer will make comparison of experiments:

In the report, the result of (b) is compared with the result of (a) and (c) placebo.

In the comparison there must be data of experiment: 
After six to ten weeks of immunization, allergic reactions to stings occurred in seven of twelve patients treated with the placebo, seven of twelve treated with crushed-body extract, and one of eighteen treated with the venom.

Now the readers can understand the main idea of the text. They can make the decision that the correct answer to Question 1 is "A".

According to the world knowledge of ordinary readers, vaccines can be injected to prevent some diseases. This text talks about the prevention of sting reactions, so the two things for injection must be something similar to vaccines. With the linguistic context located in the question, we will know the meaning of "venom" and "serum". In answering Question 2, according to the understanding of the text's main idea, it is easy to judge that " $\mathrm{B}$ ' (poison extracted from bees) is the correct answer. In answering Question 3, experience shows that vaccines should be injected before people become ill, so "A" and "C" may be chosen. There is specific time in "C" (six to ten weeks before...). This is not mentioned in the text. According to our world knowledge, the injected people may not predict the time when they will be stung by the bees. So "A" is the correct answer. Of course, this process of understand is very short in real reading. The purpose of showing it here is to explain the importance of reorganizing the context constructed by the writer in reading comprehension.

\section{Methods in English Teaching of Reading Related to Context}

In English teaching of reading, because of the differences of culture, linguistic competence and common knowledge between writers and students, it is very important to train the students' ability of reaction and reconstruction of context. Teachers may use the following methods in English teaching of reading:

(A) Choose suitable teaching materials and strengthen the students' consciousness of context. The specific method is to ask students to read a text in normal speed. After reading the teacher should make the students judge the field of discourse, tenor of discourse, mode of discourse and the main situations set up in the text. After some time the students' consciousness of context will be raised.

(B) Train the students' ability of reconstructing the context set up by the writer and the reactive ability of stimulating the common knowledge quickly. The concrete way is to ask the student to read a text in normal speed. Then the teacher immediately asks some questions connected with these abilities. It does not matter if the students give wrong answers. What they are required is to react quickly.

(C) Train the students' ability of predicting the following text quickly. The students are required to predict what will happen in the text when they begin to read the text. To make correct prediction, the students should construct the contextual factors concerned first. So training the ability of prediction should begin after the training of method (A) and (B).

(D) Enlarge the students' world knowledge. Students of English major should be encouraged to read books in other fields. Thus their common knowledge will be enlarged.

(E) The situations set up by the writer in the previous part of the text also belong to common knowledge. In reading teachers should train the students' ability of recalling the situations in the previous part of the text.

(F) Courses on the culture of English-speaking countries should be offered to reduce the cultural differences between writers and students.

(G) In setting up questions of multiple choices in reading comprehension, teachers should pay attention to the questions concerning the students' reactive ability of context.

Besides the training of contextual knowledge, vocabulary and basic linguistic knowledge are also important in understanding a text. In setting up questions of reading comprehension test, teachers should be guided by certain theories. If the questions are concerned with contextual ability, teachers should pay attention to the students' linguistic level. Teachers should also notice that the questions should be helpful to test whether the students really understand the text. For instance, if the teacher set up questions like "How many people were treated with the venom?" in Example (4), these questions will be helpless for the teacher to grasp the students' degree of comprehension because the students can get answers to these questions without reading the whole text. Question 2 of Example (4) is more helpful in testing the students' degree of understanding the whole text and their reading ability.

\section{Conclusion}

Context theory is a branch of the theory of text linguistics. The theory of context can be applied in English teaching, especially the teaching of reading. In English teaching of reading, if the teacher consciously uses context theory to guide teaching, the students will overcome the difficulties in reading comprehension more easily and they will read faster and make fewer mistakes. This paper provides some methods in the teaching of reading based on context theory. I hope teachers and students will benefit from it. 


\section{References}

Firth, J. (1951). Papers in Linguistics. Oxford: Oxford University Press.

Halliday, M. A. K. (1989). Spoken and Written Language. Oxford: Oxford University Press.

Huang, Guowen. (1997). Essentials of Text Analysis. Changsha: Hunan Education Press.

Hymes, D. (1972). On Communicative Competence. In J. Pride, \& J. Holmes (Eds.), Sociolinguistics. Harmondsworth: Penguin.

Liu, Chendan. (2000). Text Linguistics for Teachers. Shanghai: Shanghai Foreign Language Education Press.

Steffensen, M. (1986). Register, Cohesion and Reading Comprehension. Applied Linguistics, 7, 50-52.

Van Dijk, T. (1977). Text and Context: Explorations in the Semantics and Pragmatics of Discourse. London: Longman. 